\title{
La pertinencia social de la maestría en dirección estratégica empresarial de la UGB de la República de El Salvador y desde la evaluación multinacional de expertos
}

The social relevance of expertise in corporate strategic direction of the UGB of the Republic of El Salvador and from the multinational expert evaluation

Donato Vallín González

Universidad de Guadalajara

dvallin@cucsur.udg.mx

\section{Resumen}

Con la idea de conocer el impacto en la sociedad que ha tenido la maestría en dirección estratégica empresarial (MDEE) de la Universidad Gerardo Barrios (UGB), localizada en la ciudad de San Miguel, de la República de El Salvador, se aplicó la metodología de GRANA, que se basa en el contexto filosófico de las organizaciones: UNESCO, OCDE, OMC, OUI, OEA, Acuerdo de Bolonia, Banco Mundial, entre otras. Es un proceso metodológico innovador decimal. Se inicia con10 procesos para realizar el análisis de la evaluación, primero con la evaluación interna o autoevaluación a la MDEE (considerada como una mirada a este análisis del artículo) y al final con la evaluación externa (como una segunda mirada del análisis) como proceso 10 . La evaluación interna fue realizada por un comité compuesto por cinco personas asociadas al programa de la maestría en dirección estratégica empresarial (MDEE): cuatro profesores, cuatro directivos y un estudiante. Mientras que la evaluación externa, ${ }^{4}$ o segunda mirada, estuvo compuesta por seis evaluadores externos, expertos en la disciplina y provenientes de distintos países (Estados Unidos, Italia, España, Colombia y México); además, se incluyó en los procesos a dos observadores, uno de Colombia y otro de México como coordinador general de la evaluación de la organización evaluadora-acreditadora GRANA. Para los procesos y procedimientos de la evaluación se utilizó una plataforma informática llamada SIEVAS, 
que facilita el llenado de la evaluación interna y la evaluación externa, así como la interacción entre evaluadores, que contemplan significados, contextos, referencias bibliográficas, glosario, escala decimal para calificar niveles de calidad, fortalezas, debilidades, el plan de mejora permanente, formatos para integrar las estadísticas en retrospectiva y prospectiva, disponibilidad informática para incorporar documentos probatorios y sitios web que ayudan al proceso de evaluación. También cuenta con gráficos que se construyen automáticamente conforme se incorpora la evaluación a la plataforma. Este artículo tiene la finalidad de comparar las dos miradas del proceso y las conclusiones de ambas evaluaciones, vistas desde la utilidad social en los procesos formativos y el desempeño profesional de sus egresados desde su creación y oferta en el entorno regional, local, nacional e internacional.

Palabras clave: Calidad, pertinencia, SIEVAS, GRANA, evaluación interna, evaluación externa, estudios universitarios.

\section{Abstract}

With the idea of knowing the impact on society that has expertise in corporate strategic direction (MDEE) University Gerardo Barrios (UGB), located in the city of San Miguel, the Republic of El Salvador, the methodology was applied GRANA, which is based on the philosophical context of organizations: UNESCO, OECD, WTO, OUI, OAS, Bologna Agreement, World Bank, among others. It is an innovative methodological process decimal. It starts CON10 processes for the analysis of the assessment, first with internal or self-evaluation to MDEE (considered as a look at this analysis of the article) and end with the external evaluation (as a second glance analysis) as a process 10. the internal assessment was conducted by a committee composed of five persons associated with the master's program in business strategic direction (MDEE): four teachers, four directors and a student. While the external evaluation, 4 or second glance, consisted of six external experts in the discipline and evaluators from different countries (United States, Italy, Spain, Colombia and Mexico); also it was included in the processes two observers, one from Colombia and one from Mexico as general coordinator of the evaluation of the evaluation GRANA-accrediting organization. For processes and procedures for evaluating a software 
platform called SIEVAS, which facilitates filling of the internal evaluation and external evaluation, as well as the interaction between evaluators, which include meanings, contexts, references, glossary, decimal scale was used grade quality levels, strengths, weaknesses, permanent improvement plan, formats for integrating statistics retrospective and prospective, information availability to include supporting documents and websites that help the evaluation process. It also features graphics that are built automatically as the platform evaluation is incorporated. This article aims to compare the two eyes of the process and the conclusions of both evaluations, views from the social utility in educational processes and professional performance of the graduates since its inception and supply in the regional environment, local, national and international.

Keywords: Quality, relevance, SIEVAS, Grana, internal evaluation, external evaluation, university studies.

Fecha recepción: Junio $2014 \quad$ Fecha aceptación: Diciembre 2014

\section{Introducción}

Los referentes de GRANA ${ }^{1}$ se basan en los contextos filosóficos de las organizaciones UNESCO,${ }^{2}$ OCDE, ${ }^{3}$ OMC,${ }^{4}$ OUI, ${ }^{5}$ OEA,${ }^{6}$ Acuerdo de Bolonia, ${ }^{7}$ Banco Mundial, ${ }^{8}$ entre otras, asociados particularmente a procesos de evaluación en universidades e instituciones de educación superior con fines de impulsar su calidad en la oferta de programas y planes para la formación profesional. Considerando que la evaluación ofrece bases para la reflexión y la toma de decisiones, y al situar conocimientos y teorías en un contexto real, se convierte en una bisagra entre la investigación y la práctica. Los resultados sirven, por supuesto, para fundamentar decisiones, justificar solicitudes de apoyos o compartir con colegas en aras de encontrar soluciones a conflictos y problemas. La evaluación constituye un instrumento estratégico en la mejora de lo evaluado, vinculándose los diferentes enfoques y etapas que la componen, así como los motivos que la justifican. Por añadidura, se describen con sumo detalle los procedimientos de evaluación de GRANA para aquilatar 
los avances, la calidad de las estrategias y el grado de integración de las dimensiones-rubro asociadas a los contenidos académicos, que representan un valioso instrumento para la planeación del proceso de mejoramiento en lo evaluado.

Evaluar, ¿para qué?

La evaluación ocupa un nicho entre la investigación y la práctica educativas ${ }^{2}$ : constituye una base de conocimiento amplio para la toma de decisiones y facilita el tránsito de la teoría al terreno de la práctica y su aplicación emanada de una investigación para el terreno académico.

Es de particular relevancia que la investigación educativa pueda aportar elementos para la práctica y la ejecución de políticas y estrategias educativas. Mientras que el investigador parte de una hipótesis y busca posteriormente un terreno propicio para la comprobación de esta, la evaluación, en cambio, parte de la identificación de un grupo y una entidad específicos, como un curso, un departamento o una institución. Este grupo o entidad, como objeto de evaluación, pasa a ser analizado por medio de criterios previamente seleccionados, y a la luz de cualidades y objetivos deseados. La evaluación se distingue también de la investigación por la manera en que se manejan las diferentes perspectivas. Los evaluadores suponen que su trabajo tiene un valor, tal como está implícito en el mismo término evaluación. El evaluador se acerca al objeto en términos de lo que es deseable, tomando como punto de partida uno o varios puntos de vista. Una evaluación comprensiva utiliza una base de información lo más amplia posible, con objeto de presentar los resultados desde varias perspectivas, y se realiza por medio de la recopilación y el análisis de la información a partir de un abanico de actores. En la educación global, por ejemplo, el actor puede ser una persona o una entidad interesada en el proceso de internacionalización. ${ }^{9}$ Una vez realizada la evaluación, partiendo de diferentes puntos de vista, el encargado de llevarla a cabo hace juicios y recomendaciones desde los observadores externos. En el ámbito administrativo, la evaluación se utiliza para tomar decisiones sobre problemas reales, tratando de adoptar las mejores soluciones posibles en función de la información disponible en el momento. En una institución que haya incluido la internacionalización en su misión, a los académicos les corresponde tomar decisiones sobre la manera de internacionalizar sus cursos. Los administradores, por su parte, enfrentan el reto de preparar estudiantes dentro de un marco de criterios académicos y de acreditación ya establecidos. 
En cualquier caso, los educadores o administradores internacionales necesitan información para tomar las decisiones apropiadas. La evaluación es particularmente útil para los administradores en el momento de planear y diseñar políticas y programas institucionales. La planeación estratégica constituye un resultado natural de la evaluación, y no debe limitarse al ámbito institucional; se recomienda que tome en cuenta el entorno en que se halla inmersa la institución. Las recomendaciones resultantes de la evaluación pueden ser utilizadas para el cambio: el mejoramiento de ciertos rubros y sectores, el apoyo de ciertos grupos de interés, el ahorro de recursos o la realización de cualquier otro tipo de objetivos planteados. En suma, de las recomendaciones puede surgir la planeación de un proceso de cambio o medidas de mejoramiento. La evaluación sirve, además, como fuente de información estratégica para quienes toman las decisiones y para la implantación de políticas y procedimientos que redundarán en beneficio de diversos actores. Existen, también, preguntas características a las que se enfrentan las personas que tienen la responsabilidad de enseñar políticas educativas y que la evaluación puede ayudar a responder, por ejemplo, cómo pueden ser cumplidas las demandas del sector académico y administrativo, qué datos son necesarios para encauzar demandas y reclamos, o bien para apoyar argumentos y requerimientos de financiamiento de la formación. En este contexto y con la idea de conocer el impacto en la sociedad que ha tenido la maestría en dirección estratégica empresarial (MDEE), ${ }^{9}$ se aplicó la metodología de GRANA para realizar las evaluaciones interna y externa. La evaluación externa fue realizada con seis evaluadores, ${ }^{10}$ provenientes de Estados Unidos (2), Italia (1), España (1), Colombia (1) y México (1); la evaluación interna fue realizada por profesores y directivos de la UGB.

\section{Contexto de la UGB ${ }^{11}$}

La Universidad Gerardo Barrios fundamentada en la docencia, la investigación y la proyección social; tiene como objetivo proyectar el quehacer universitario hacia el entorno por medio de la aplicación y generación de conocimientos que sean socialmente útiles y que contribuyan a los avances científicos, técnicos y culturales en la zona oriental de El Salvador para incidir en el mejoramiento de la calidad de vida de los individuos y en la formación integral de la sociedad. La UGB cuenta con dos sedes: la sede de San Miguel y la sede de Usulután. En estas sedes se realizan diversas acciones académicas que son 
determinadas en las líneas de acción plasmadas en la política de proyección social, las que surgen de necesidades planteadas por las comunidades mismas a través de diagnósticos, por solicitud de organizaciones estatales, municipales y no gubernamentales. La política de proyección social establece que se realizarán proyectos en las áreas académicas de derecho, educación, salud, administración y economía, tecnología, ingeniería civil, arte y arquitectura, las cuales contienen las siguientes líneas básicas de acción: asistencia jurídica en materia de familia, laboral, civil y penal; equidad en la educación; problemas sociales; asistencia técnica en administración, mercadeo y ventas a las pymes; software de uso aplicativo en diferentes ámbitos; apoyo técnico en materia de ingeniería civil; planificación territorial.

Actualmente la UGB ocupa el undécimo lugar a nivel nacional, de acuerdo con la tabla estadística 1. 


\begin{tabular}{|c|c|c|c|c|c|c|c|}
\hline Ranking & $\frac{\text { Ranking }}{\underline{\text { Mundial }}}$ & $\underline{\text { Universidad }}$ & Det. & $\begin{array}{l}\text { Presencia } \\
\text { (Posición*) }\end{array}$ & $\begin{array}{l}\text { Impacto } \\
\left.\text { (Posición }{ }^{*}\right)\end{array}$ & $\begin{array}{l}\text { Apertura } \\
\left.\text { (Posición } n^{*}\right)\end{array}$ & $\begin{array}{l}\text { Excelencia } \\
\text { (Posición*) }\end{array}$ \\
\hline 1 & 3157 & $\underline{\text { Universidad de El Salvador }}$ & 표 & 566 & 4870 & 1228 & 4116 \\
\hline 2 & 4027 & $\underline{\text { Universidad Francisco Gavidia }}$ & 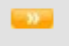 & 1598 & 3944 & 989 & 5484 \\
\hline 3 & 4060 & $\begin{array}{l}\text { Universidad Centroamericana José Simeón } \\
\underline{\text { Cañas }}\end{array}$ & Ex & 3639 & 4656 & 2191 & 4892 \\
\hline 4 & 6337 & $\underline{\text { Universidad Don Bosco El Salvador }}$ & 피 & 962 & 10557 & 3083 & 4892 \\
\hline 5 & 6682 & $\begin{array}{l}\text { Instituto Especializado de Formación } \\
\text { Diplomática IESFORD }\end{array}$ & 필 & 15383 & 2902 & 17244 & 5484 \\
\hline 6 & 7228 & $\underline{\text { Universidad Tecnológica de El Salvador }}$ & (2) & 5408 & 9805 & 910 & 5484 \\
\hline 7 & 8091 & $\underline{\text { Universidad Dr José Matias Delqado }}$ & w & 982 & 11317 & 2535 & 5484 \\
\hline 8 & 10738 & $\underline{\text { Universidad Luterana Salvadoreña }}$ & Eiv & 9519 & 10799 & 10908 & 5484 \\
\hline 9 & 10854 & $\underline{\text { Universidad de Oriente El Salvador }}$ & 피 & 4616 & 13941 & 3073 & 5484 \\
\hline 10 & 11625 & $\underline{\text { Universidad Evangélica de EI Salvador }}$ & Ex & 14195 & 14115 & 12534 & 4565 \\
\hline 11 & 12730 & $\underline{\text { Universidad Gerardo Barrios }}$ & Ex & 4253 & 14276 & 13346 & 5484 \\
\hline 12 & 12885 & $\underline{\text { Universidad Católica de EI Salvador }}$ & 피 & 2331 & 14956 & 13541 & 5484 \\
\hline 13 & 12951 & Escuela Superior de Economia y Neqocios & En & 12415 & 12019 & 16154 & 5484 \\
\hline 14 & 13332 & $\underline{\text { Universidad Politécnica de EI Salvador }}$ & 피 & 9278 & 13769 & 13870 & 5484 \\
\hline 15 & 14194 & $\begin{array}{l}\text { Academia Nacional de Sequridad Pública } \\
\text { República de El Salvador }\end{array}$ & 페 & 7777 & 17028 & 4381 & 5484 \\
\hline 16 & 14501 & Instituto Tecnológico Centroamericano & 필 & 2178 & 16757 & 14617 & 5484 \\
\hline 17 & 15860 & Universidad Autónoma de Santa Ana & Ev & 9856 & 16970 & 14027 & 5484 \\
\hline 18 & 16196 & $\underline{\text { Universidad Salvadoreña Alberto Masferrer }}$ & 팔 & 16225 & 16000 & 15800 & 5484 \\
\hline 19 & 16622 & $\underline{\text { Universidad Pedaqóqica de El Salvador }}$ & w & 12934 & 17403 & 14105 & 5484 \\
\hline 20 & 16832 & Escuela de Comunicación Mónica Herrera & $m$ & 16647 & 16479 & 16868 & 5484 \\
\hline
\end{tabular}

Tabla estadística 1. Escala web universidades de El Salvador

La UGB cuenta con cinco facultades: Ciencias Jurídicas, Ciencias y Humanidades, Ciencias Empresariales, Ciencia y Tecnología, Ingeniería Civil y Arquitectura.

La UGB, en convenio con el Ministerio de Educación de El Salvador, desarrolló el plan de vida 2008-2014, beneficiando a una población acumulada de 23934 estudiantes de educación media de la zona oriental, con el objetivo de contribuir con la formación integral de jóvenes, promoviendo valores y actitudes positivas en temas sobre derecho humanos, construcción de su propio proyecto de vida y orientación vocacional. Actualmente han sido beneficiadas 14 instituciones de educación media del departamento de Usulután, con una población beneficiaria de 2213 estudiantes.

El objetivo de la UGB es contribuir al desarrollo económico y social de El Salvador por medio de la formación integral de profesionales con los conocimientos, habilidades, 
actitudes y valores necesarios para enfrentar con éxito los retos del mundo empresarial. El contexto nacional y mundial en que se desarrollan los negocios cambia vertiginosamente, y la competitividad se ha convertido en un atributo necesario para las empresas de todo tamaño. Ante tales exigencias, la Facultad de Ciencias Empresariales ofrece las siguientes carreas: licenciatura en administración de empresas, técnico en contaduría pública, técnico en mercadeo y ventas y la maestría en dirección estratégica empresarial (MDEE) con el objetivo de formar profesionales competitivos que el mundo de los negocios necesita, bajo un modelo educativo por competencias y un equipo docente altamente calificado, y en los recursos educacionales y tecnológicos que facilitan el proceso de enseñanza-aprendizaje. La historia de tres décadas está representada por miles de profesionales que actualmente laboran para importantes empresas e instituciones o se han convertido en empresarios exitosos que le aportan dinamismo a la economía del país. La propuesta de la UGB es darle a El Salvador un legado educativo histórico representado en una sociedad más justa y humana, en la cual el bienestar económico y social sea posible para los salvadoreños.

El programa de la MDEE es relevante académicamente y responde a necesidades locales, regionales, nacionales e internacionales. El programa ha definido un proyecto educativo coherente con el proyecto institucional, en el cual se señalan los objetivos, los lineamientos básicos del currículo, las metas de desarrollo, las políticas y estrategias de planeación y evaluación y el sistema de aseguramiento de la calidad. El programa es de dominio público. Toda la información anteriormente referida está integrada en el SIEVAS ${ }^{12}$ y fue corroborada durante las visitas y entrevistas realizadas a la UGB por los evaluadores externos. La evaluación interna y externa del rubro "Impacto social de la formación" se desarrolló con los siguientes ítems: 1.1. Coherencia entre la visión, la misión y los objetivos planteados en la génesis de la formación con los resultados actuales; 1.2. Participación de entes internos y externos a la institución en la planeación; 1.3. Pertinencia y competitividad en el ámbito nacional e internacional; 1.4. Reconocimiento de la sociedad a los egresados por su desempeño; 1.5. Competitividad de los egresados ante similares externos; 1.6. Percepción de la comunidad científica, colegios especializados, egresados y empleadores; 1.7. Impacto en la inserción laboral nacional e internacional; 1.8. Percepción de los estudiantes, profesores y personal administrativo; 1.9. Egresados inscritos en el posgrado; 1.10. Participación de egresados en la mejora de la formación. Fueron aplicados 
para esta evaluación, iniciando con la evaluación interna, siguiendo con la evaluación externa de pares expertos en la disciplina y la entrega de resultados. Ambas evaluaciones se realizaron con la metodología de GRANA, utilizándose la plataforma informática del SIEVAS. Los evaluadores externos expertos fueron de cinco países: Estados Unidos, Colombia, Italia, España y México. El objetivo del artículo se centra en el análisis entre las evaluaciones interna y externa con el propósito de ver concordancias y diferencias en un entorno por una parte local y por otra multinacional, además de proponer un plan de mejora permanente.

\section{Metodología}

Para realizar este trabajo fue necesario analizar los procesos, los procedimientos y los resultados obtenidos en la evaluación al programa académico de la MDEE que se desarrollan a través de la metodología de GRANA. ${ }^{7}$ Este modelo se lleva acabo de acuerdo con los siguientes momentos:

Conformación del Comité de Evaluación Interna (CEI). Se integra por un equipo de trabajo compuesto al menos por cinco personas: dos profesores, dos directivos y un estudiante. Los egresados y empleadores asociados al programa académico a evaluar son considerados en esta evaluación.

Asignación de claves al CEI para acceso a la plataforma y sistema de evaluación SIEVAS.

Capacitación en línea y roles del sistema SIEVAS. El sistema SIEVAS cuenta con una plataforma informática que da soporte tecnológico al proceso de evaluación interna, externa y de mejoramiento permanente a los programas (PA) evaluados o a evaluar. Sus servidores se localizan en Phoenix, Arizona, y cuentan con un vasto campo de almacenaje y seguridad para el hospedaje y protección de la información que es colocada durante los procesos de evaluación. Los roles del sistema SIEVAS son:

1. Administrador del sistema. Tiene acceso a la funcionalidad completa del sistema, además de ser encargado del ingreso de la información correspondiente a la parametrización, la creación de usuarios, programas e instituciones a evaluar. 
2. Evaluador interno. Es quién debe dar cuenta de la calidad del programa o institución evaluada; para ello deberá dar respuesta a cada uno de los ítems, identificando fortalezas, debilidades, acciones de mejoramiento y documentos soportes de la información ingresada, y presentar la información estadística en los formatos dispuestos.

3. Evaluador externo. Evaluación hecha por pares externos teniendo como referencia los resultados obtenidos en la evaluación interna.

4. Experto. Consiste en llevar un registro de la experiencia de personas expertas en las diferentes áreas de conocimiento, y que se pueda tener como referencia de acuerdo a los temas de interés, accediendo a un foro que se jerarquiza por áreas y núcleos de conocimientos.

5. Consultor. Es un usuario que podrá ver la información ingresada al sistema, pero solo a manera de consulta, es decir, que no podrá escribir ni modificar la información ingresada por los responsables de las evaluaciones.

6. Sistema decimal de calidad. Para facilitar los procesos de evaluación, GRANA conformó un sistema métrico decimal para la medición de la calidad, denominado sistema Vallín, que consiste en 10 rubros-dimensiones, 100 ítems (10 ítems por 1 rubro-dimensión) y 1000 indicadores de segunda y tercera generación (10 indicadores por ítem). La escala de calidad es decimal ( $0-10$, donde 0 es calidad nula y 10 es excelente). Son 10 los procesos a realizar y 100 procedimientos a lo largo de la evaluación. En tiempo, son 100 días promedio para cada proceso de evaluación. Son 10 tablas o formatos para la elaboración estadística de la evaluación.

7. Modelo de evaluación circular y cíclica. El modelo de evaluación de GRANA es actualizado permanentemente bajo un esquema circular y cíclico, como se muestra en la figura 1. Este modelo aplica a su vez en los procesos de mejoramiento permanente (PMP) de las entidades evaluadas de las instituciones de educación, como en los programas académicos. 


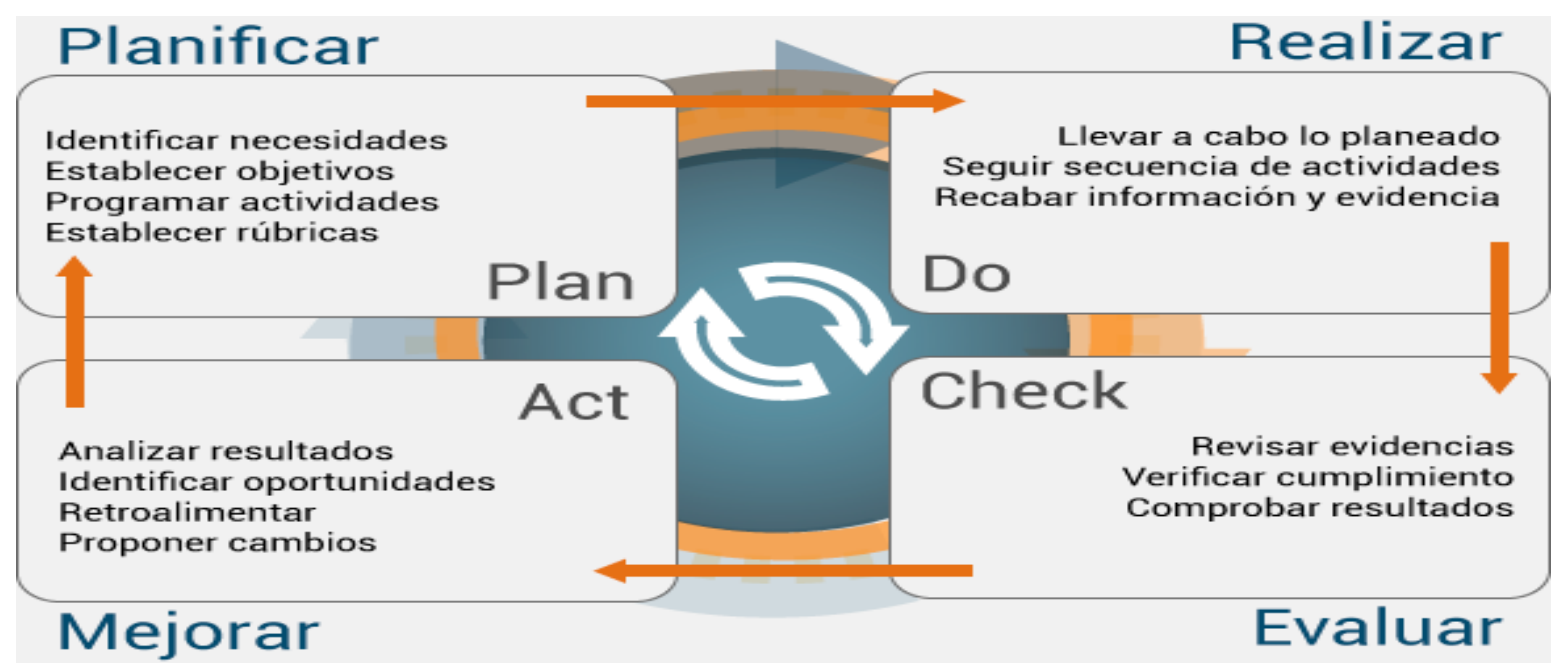

Figura 1. Modelo de evaluación GRANA, cíclico y circular.

Evaluación interna (EI). Se inicia con la integración y sistematización de la información referida a la unidad objeto de evaluación. El Comité de Evaluación Interna (CEI) integrará al SIEVAS la información de la EI, mediante las observaciones, opiniones y valoraciones generadas a lo largo del proceso. Esta evaluación consiste en que el CEI construya puntual y objetivamente:

a) Integración del FODA del PA.

b) Integración de información estadística del PA.

c) Construcción del plan de mejora permanente (PMP) del PA.

d) Integración de documentos probatorios del FODA y datos estadísticos del PA. Toda esta información es integrada al SIEVAS, como se observa en la figura 2. 


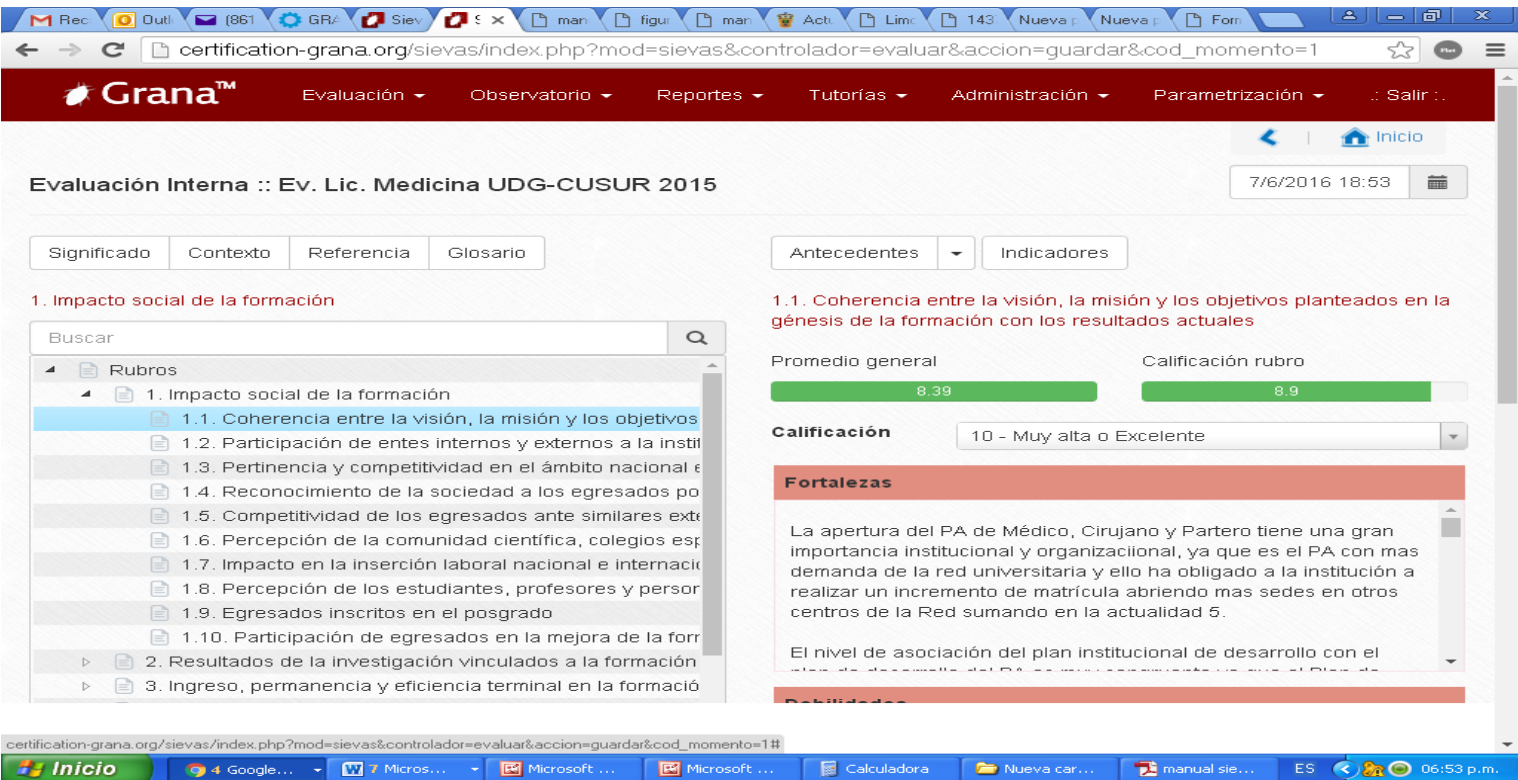

Figura 2. Vista de la plataforma SIEVAS.

Selección de evaluadores externos (EE). Se analizan perfiles requeridos de académicos destacados en la disciplina a evaluar, con conocimiento y experiencia en evaluación académica. Los EE serán de distintos países y al menos de dos continentes.

Evaluación externa en línea. Se realiza mediante la plataforma SIEVAS; en ella se plasman las coincidencias y diferencias del análisis previo a la visita in situ a la institución evaluada.

Visita a las instalaciones de los evaluadores externos. Para verificar información y realizar entrevistas a los principales involucrados en la evaluación, se realiza una visita de tres días a las instalaciones de la institución evaluada.

Entrega de predictamen a la institución. Al final de la visita es entregado un preinforme a la institución evaluada.

\section{Reporte-dictamen de resultados de la evaluación y plan de mejora permanente.} Después de la visita a la institución y en un plazo no mayor a 30 días, los evaluadores externos entregan el reporte-dictamen sobre el proceso de evaluación. En este reporte se describen las recomendaciones para la mejora permanente del PA evaluado.

Metaevaluación del proceso. El proceso también es evaluado por la institución evaluada para la mejora del modelo y el sistema SIEVAS de GRANA. 
Sobre el sistema y los criterios de evaluación de GRANA y del SIEVAS. La escala de medición es cualitativa y cuantitativa, y se refiere a niveles de capacidad, competitividad, pertinencia, cobertura e innovación del programa o institución; en ella se establecen los valores de calidad asociados al juicio de valor otorgado a cada ítem y al resultado de calidad obtenido una vez finalizada la evaluación. La escala definida es la siguiente: $0=$ Nulo, 1=Muy escasa, 2=Escasa, 3=Medianamente escasa, 4=Poco incipiente, 5=Medianamente incipiente, 6=Incipiente, $7=$ Incipientemente alta, 8=Medianamente alta, 9=Alta, 10=Muy alta o excelente. Así se observa en tabla 1.

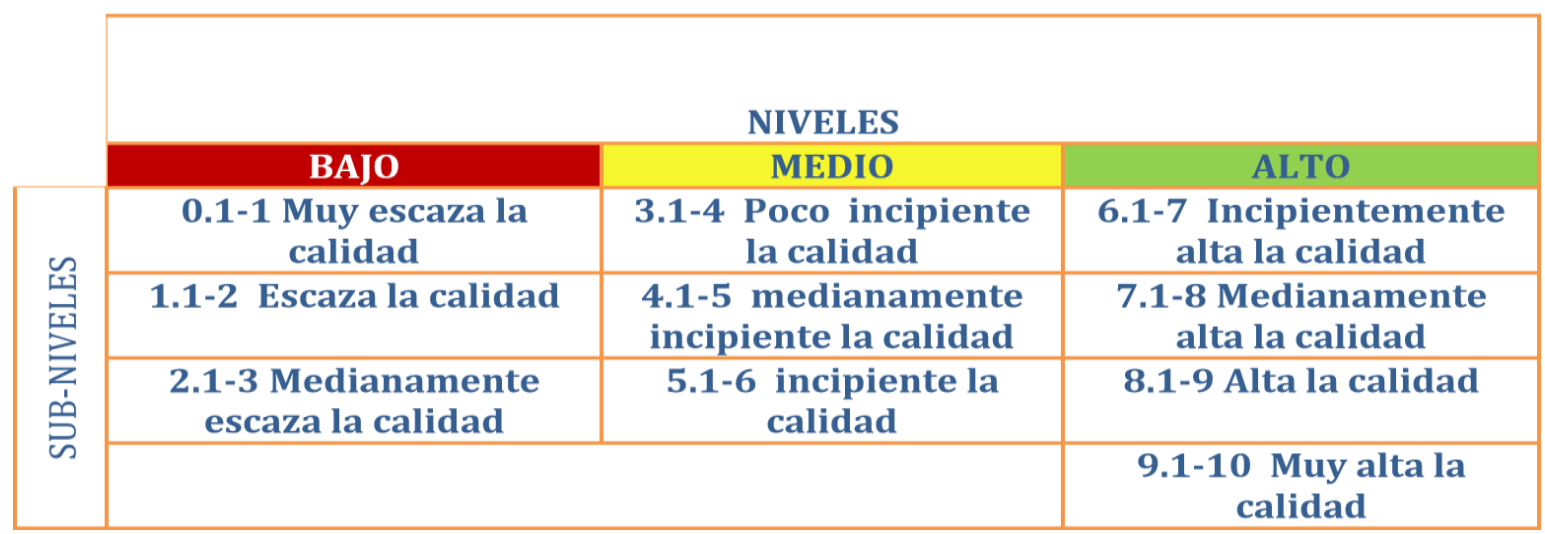

Tabla 1. VERDE (alta la calidad), rango de calificación entre 6.1-10. AMARILLO (media la calidad), rango de calificación entre 3.1-6. ROJO (baja la calidad), rango de calificación entre 0-3

El SIEVAS cuenta con gráficos dinámicos que permiten visualizar en línea y en tiempo real el nivel de calidad con que las evaluaciones interna y externa del PA van desarrollándose, como se muestra en la gráfica1: el círculo de color rojo al centro representa el nivel bajo en la calidad, el círculo amarillo céntrico representa el nivel medio de calidad y el último círculo, de color verde, representa el nivel alto de calidad; la sección de color azul representa las fortalezas del PA evaluado, mientras la zona no cubierta de azul son las oportunidades del PA. 


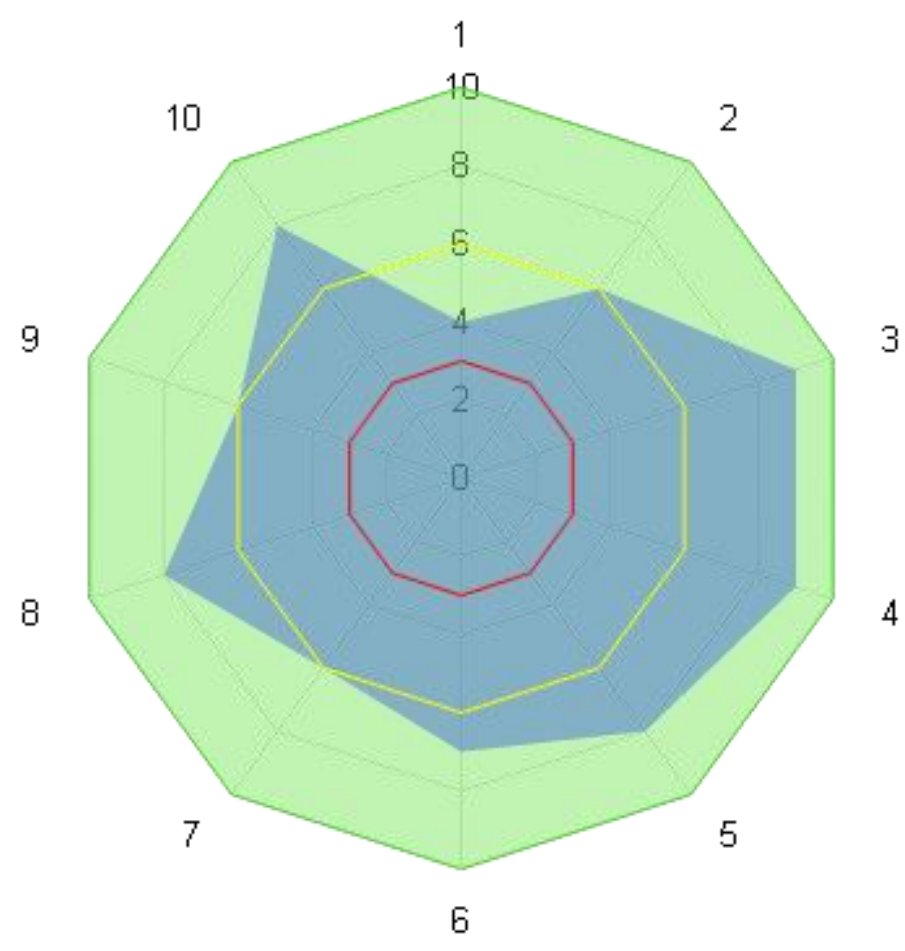

Gráfica 1. La calidad en gráfica del modelo GRANA.

\section{Resultados}

\section{Composición de los comités evaluadores}

La composición del CEI fue integrada por: Carlos Enrique Mendoza Flores, decano de la Facultad de Ciencias Empresariales y coordinador del Comité Evaluador Interno del CEI; los profesores Edgar Humberto Maravilla Carballo, Douglas Watemoth Cortez Vigil, Oscar René Rendón González, Armida Cecilia Granillo Córdova, José William Roldán Granados y Bayron Adrián Meléndez Rubio, director de calidad de la UGB. Mientras que la conformación del CEE fue integrada por: Donato Vallín González, director general de GRANA-ISTEC; Adolfo Espinoza de los Monteros Cárdenas, de la Universidad de Guadalajara, México, quien fungió como coordinador de la evaluación académica externa (CEE); Douglas Thomas, profesor y director del programa de dirección estratégica y negocios internacionales de la Universidad de Nuevo México, con sede en Alburquerque, Nuevo México, Estados Unidos; Andrea Giuntini, profesor especializado en la historia de 
la economía, vinculado al Departamento de Economía de la Universidad de Modena, Italia; María Claudia Aponte González, consultora en planeación e internacionalización de instituciones educativas de Medellín, Colombia; Federico Martínez-Carrasco Pleite, profesor del Departamento de Economía Aplicada de la Universidad de Murcia, España; Germán López de la Madrid, consultor de calidad e internacionalización de instituciones educativas, Universidad de Utah, Estados Unidos.

\section{La metodología aplicada}

La metodología aplicada para el presente artículo se realiza en los siguientes ítems: 1. Coherencia entre la visión, la misión y los objetivos planteados en la génesis de la formación con los resultados actuales, 2. Participación de entes internos y externos a la institución en la planeación, 3. Pertinencia y competitividad en el ámbito nacional e internacional, 4. Reconocimiento de la sociedad a los egresados por su desempeño, 5. Competitividad de los egresados ante similares externos, 6. Percepción de la comunidad científica, colegios especializados, egresados y empleadores, 7. Impacto en la inserción laboral nacional e internacional, 8. Percepción de los estudiantes, profesores y personal administrativo, 9. Egresados inscritos en el posgrado, 10. Participación de egresados en la mejora de la formación.

\section{La evaluación externa}

Posteriormente se realizó el análisis con los evaluadores externos, basado en la escala cualitativa de GRANA y generando los siguientes resultados sobre sus fortalezas, debilidades y propuestas de plan de mejora para cada uno:

\section{Coherencia entre la visión, la misión y los objetivos planteados en la génesis de la formación con los resultados actuales}

\section{Fortalezas}

Los objetivos planteados por la maestría en dirección estratégica de empresas de la UGB son de interés social y coherentes con la visión y misión planteadas, habiendo sido revisados mediante un proceso participativo a los dos años de la creación del título. 


\section{Debilidades}

El proceso de revisión de la misión y visión de la titulación ofertada y de los objetivos planteados en su génesis no se acredita con datos o informaciones objetivos de adecuación y de logro de objetivos propuestos.

\section{Plan de mejoramiento}

Plantear indicadores y procedimientos sistemáticos de seguimiento con los que poder acreditar y valorar el nivel de consecución de los objetivos propuestos por la titulación para los próximos años.

\section{Puntaje: 7.}

\section{Participación de entes internos y externos a la institución en la planeación}

\section{Fortalezas}

En el proceso de planeación del título de máster, revisado recientemente, participaron representantes de la decanatura de la titulación de Ciencias Empresariales, docentes del cuerpo académico del programa -con experiencia laboral en el ámbito- y un consultor empresarial externo.

\section{Debilidades}

La participación en la planeación dentro de la titulación de expertos externos, egresados y actores de la zona es incipiente y reducida.

\section{Plan de mejoramiento}

Dar participación en próximas revisiones y procesos de planeación del título a representantes de cuerpos colegiados y profesionales, y egresados que desarrollan su actividad profesional, realizándose mediante un proceso sistemático de acreditación de esos procesos, con la identificación de sus participantes y sus propuestas.

\section{Puntaje: 7.}




\section{Pertinencia y competitividad en el ámbito nacional e internacional}

\section{Fortalezas}

El título de máster ofertado es pertinente, siendo la temática de esta formación de posgrado

justificada, aportando formación universitaria de especialización para recién licenciados y para profesionales o gerentes de empresas y organizaciones, siendo destacado su enfoque docente dirigido al desarrollo de competencias profesionales.

\section{Debilidades}

No se ha acreditado la pertinencia de esta formación educativa, en términos de la existencia de una demanda o un déficit formativo en su zona de influencia, ni tampoco un análisis de la oferta formativa de posgrado que sobre su temática existe a nivel regional, nacional o en países del entorno más próximo.

\section{Plan de mejoramiento}

Realizar un diagnóstico de la demanda social de esta formación de máster, de la presencia de titulaciones de posgrado de igual temática en el ámbito nacional e internacional y de los elementos competitivos (en términos de calidad, facilidades de acceso, de situación geográfica, de especialización temática o de calidad) de que dispone el título ofrecido.

\section{Puntaje: 0.}

\section{Reconocimiento de la sociedad a los egresados por su desempeño}

\section{Fortalezas}

Si bien es inicial el proceso de recopilación de reconocimientos sociales de egresados de la titulación, dado lo reciente de esta titulación, se cuenta con registros de varias participaciones de estos en eventos científicos y redes temáticas en el ámbito de estudio del título, así como de indicios de una adecuada valoración por parte de empleadores y egresados, proxy del reconocimiento social de sus egresados.

\section{Debilidades}

No se cuenta con un registro de seguimiento de la labor desarrollada por los egresados de la titulación, así como de los reconocimientos por estos recibidos en su labor profesional.

\section{Plan de mejoramiento}

Desarrollar mecanismos de registro y seguimiento de la labor profesional de los egresados, así como de sus estudiantes durante el periodo de formación recibida o previa a su ingreso, 
implementando un proceso de conexión permanente y comunicación directa con estos, a través de la plataforma virtual de la universidad, de redes sociales y profesionales o con la creación de una asociación de antiguos alumnos.

Puntaje: 8.

\section{Competitividad de los egresados ante similares externos}

\section{Fortalezas}

El diseño de la titulación bajo un enfoque de enseñanza basado en el desarrollo de competencias, con el estudio en su currículo de casos prácticos reales y de empresas del entorno, aporta valiosas herramientas para una adecuada inserción en el mercado laboral, siendo positiva la valoración que de la formación recibida y de sus egresados tienen los empleadores y egresados con los que tuvo un encuentro durante el proceso de evaluación externa de la titulación.

\section{Debilidades}

No se cuenta con un registro ni con estadísticas del nivel de inserción en el mercado laboral de los egresados del máster, que permita conocer su nivel de empleabilidad y las ventajas competitivas que esta formación les aportó en su acceso al mercado laboral y/o el desarrollo de su labor profesional frente a otros profesionales.

\section{Plan de mejoramiento}

Promover un sistema de seguimiento de los egresados de la titulación en términos de su inserción al mercado laboral y el desarrollo de sus carreras profesionales, proponiéndose el envío de manera sistemática cada dos años de cuestionarios a antiguos alumnos y a empleadores para recabar dicha información, manteniendo una base de datos que permita aportar cifras y valoraciones del nivel de competencia de los egresados de la titulación frente a los de otras universidades o centros.

\section{Puntaje: 8.}




\section{Percepción de la comunidad científica, colegios especializados, egresados y empleadores}

\section{Fortalezas}

La valoración de egresados y empleadores que se alcanzó con las entrevistas realizadas durante el proceso de evaluación externa del título permitió comprobar indicios de una adecuada valoración de la comunidad acerca de esta titulación. Además, los estudiantes de la maestría realizan proyectos sociales y de investigación sobre problemas concretos de la sociedad, en diferentes organizaciones de la zona, entregando a la finalización del mismo los documentos finales elaborados, contribuyendo estas prácticas a aumentar la valoración y conocimiento de la maestría entre actores sociales de su zona de influencia.

\section{Debilidades}

No se cuenta con información objetiva de la percepción de actores externos (empleadores, colegios profesionales o egresados) acerca de la titulación y la labor formativa e investigación aplicada desarrollada en la misma, salvando las entrevistas realizadas por el grupo de evaluadores externos a algunos empleadores y egresados, desconociéndose la valoración de otros actores sociales y empresariales de la zona de influencia.

\section{Plan de mejoramiento}

Desarrollar sistemas de recolección de la valoración y percepción social que empleadores, egresados y otros actores sociales o empresariales tienen de la titulación, implementando mecanismos de recolección sistemática de información, así como mecanismos para evaluar el nivel de satisfacción y valoración del interés de los trabajos realizados por miembros de la titulación en cualquier ámbito (prácticas sociales, prácticas en empresa, realización de informes, desarrollo de servicios, etcétera).

\section{Puntaje: 5}

\section{Impacto en la inserción laboral nacional e internacional}

\section{Fortalezas}

La mayor parte de los alumnos matriculados en esta maestría son profesionales con algunos años de experiencia en el mundo laboral, siendo este un espacio de formación permanente válido para su promoción y especialización. 


\section{Debilidades}

No se cuenta con datos sobre el alumnado que ha ingresado y egresado de la maestría, ni sobre su proyección profesional en el ámbito nacional e internacional, o con informes que valoren el impacto real que el estudio de esta maestría tiene para la promoción laboral de estudiantes que ya se encuentran trabajando, o de alumnos recién licenciados en su acceso por primera vez al mercado laboral.

\section{Plan de mejoramiento}

Establecer sistemas de recogida de información sistemática y de comunicación permanente con egresados de la maestría, para conocer la evolución profesional de los estudiantes que ya se encuentran en el mercado laboral o de recién egresados de licenciaturas.

\section{Puntaje: 5.}

\section{Percepción de los estudiantes, profesores y personal administrativo}

\section{Fortalezas}

Los resultados obtenidos en las entrevistas mantenidas por el grupo evaluador externo con estudiantes de la maestría y con personal administrativo de la UGB ofrecen indicios de una alta valoración de la titulación de máster por parte de sus estudiantes y egresados, siendo elevada la implicación del profesorado de la Facultad con este título, y elevado el nivel de experiencia profesional de los profesores a tiempo parcial que imparten dicho título de posgrado, así como el nivel de compromiso del personal administrativo de la Universidad. En todas las áreas y dependencias de la Universidad se cuenta con buzones de recogida de sugerencias.

\section{Debilidades}

El alumnado plantea la existencia de algunos espacios de mejora en la docencia recibida en alguna materia. El profesorado plantea algunos espacios de mejora en los ámbitos de la coordinación y el acceso a más medios para un mejor desarrollo de su actividad docente, y de manera particular, la falta de implicación en tareas de organización docente y participación de profesores de la maestría debido a que son profesores de tiempo parcial en su mayor parte. Se han de mejorar los documentos de evaluación existentes, relativos a la valoración global de la titulación y de la calidad de la docencia que reciben. 


\section{Plan de mejoramiento}

Establecer y mejorar los procedimientos existentes de evaluación y medición -cuantitativa y cualitativa- de la calidad de la docencia, del profesorado y de los servicios que presta el conjunto de la UGB, mediante la elaboración de documentos de evaluación de la calidad que reúnan información de cuestionarios de evaluación del alumnado -por asignatura, del conjunto de la titulación y de los servicios prestados-, así como del profesorado y del personal de administración, incluyendo en todos los ítems escalas de valoración únicas. Para ello sería conveniente el desarrollo de un servicio y unidad específica de evaluación de la calidad dentro de la Universidad Gerardo Barrios, y de órganos de control de la calidad dentro de la maestría, acreditándose esos procesos de coordinación docente y de seguimiento de calidad de la formación y los servicios administrativos de la Universidad.

\section{Puntaje: 9.}

\section{Egresados inscritos en el posgrado}

\section{Fortalezas}

El programa de maestría en dirección estratégica de empresas de la Universidad Gerardo Barrios desarrolla las competencias en el ámbito del desarrollo de la actividad empresarial, como una formación válida que permite continuar estudios de doctorado en las áreas de finanzas, economía, mercadeo, recursos humanos y planeación estratégica, si bien su enfoque es de carácter profesional y no investigador.

\section{Debilidades}

No se cuenta en la Universidad Gerardo Barrios con un programa de doctorado en ninguna disciplina, ni con convenios específicos para que alumnos de la maestría puedan acceder de manera directa o con facilidades burocráticas a estudios de doctorado existentes en otras universidades nacionales, tampoc cuenta la propia maestría con profesores con ese grado académico y orientación investigadora.

\section{Plan de mejoramiento}

Procurar procedimientos para fomentar que egresados de la maestría y docentes de la Universidad puedan acceder a cursos de doctorado de universidades nacionales o internacionales, incorporando profesores con el grado de doctor, visitantes o permanentes, 
procurando, mediante convenios específicos con universidades nacionales y extranjeras, mecanismos para facilitar que alumnos de la maestría puedan acceder a programas de doctorado vinculados con la UGB, implantando mecanismos de seguimiento de alumnos de máster que accedieron posteriormente a cursos de doctorado. Se plantea la posibilidad en futuras reformas del plan de estudios del título el incorporar asignaturas o itinerarios investigadores, que dieran la formación precisa y un acceso más directo a otros programas de doctorado, que posteriormente permitan instaurar cursos de doctorado propios de la UGB o en colaboración con otras universidades.

\section{Puntaje: 3.}

\section{Participación de egresados en la mejora de la formación}

\section{Fortalezas}

La maestría dentro dell diseño del plan de estudios tuvo la participación de cuatro profesionales con diversas maestrías de corte empresarial, cada uno de ellos específicamente con un máster en dirección estratégica de empresas. Además, son ya varios los profesores de la Facultad que han realizado este, circunstancia que aporta elementos de conocimiento e implicación académica y personal a los procesos de mejora.

\section{Debilidades}

La participación directa de egresados del máster en la mejora de la formación es incipiente, en especial entre aquellos con una actividad laboral fuera de la UGB. No se cuenta con información de la participación de egresados del máster en los procesos de formación y su participación en prácticas profesionales de alumnos del título.

\section{Plan de mejoramiento}

Diseñar estrategias que permitan una participación directa de egresados y graduados en los procesos de actualización del programa de la maestría, así como en los propios procesos de docencia práctica y aplicada, apoyándose y valiéndose de su experiencia profesional tras la realización del máster, con una participación de los mismos en jornadas, conferencias o prácticas por ellos impartidas.

\section{Puntaje: 5.}




\section{Análisis, discusión y consenso en la visita y entrevistas en la UGB}

El proceso de mejoramiento permanente (PMP) es considerado como un elemento fundamental en los planes estratégicos de desarrollo de la institución en pro de la verdadera calidad académica y profesional que se requiere para impulsar la capacidad y competitividad de la región de vinculación con la UGB. Deberá ser permanente y llevarse a cabo en intervalos, con una evaluación constante en los logros de sus metas, es decir, el compromiso de los entes involucrados en el proceso formativo del futuro profesional será decisivo en el cumplimiento del PMP.

El plan vigente de la maestría en el año 2006 contaba con objetivos que reflejaban el pensamiento filosófico de la institución, lo que indica que el plan se diseñó de acuerdo con esa filosofía. Dichos objetivos obedecían a la realidad del momento y a la coyuntura socioeconómica de la zona oriental de El Salvador, que es donde la universidad tiene su mayor referente en la formación de sus profesionales. La universidad ha ido creciendo dentro de este ámbito, concretizando su vocación por la formación y en la búsqueda de mejoras continuas. Para el año 2015 se actualizó el plan de la carrera y sus objetivos siguen guardando relación con la visión y misión institucional vigente a esa fecha; un elemento importante es que el plan se elaboró de acuerdo con el enfoque por competencias. Este enfoque enfatiza en un perfil profesional con competencias generales y específicas que le permitirán al egresado afrontar los desafíos profesionales y personales. Para los siguientes años, la institución realizará un proceso participativo de revisión y actualización de la filosofía institucional, dando como resultado una nueva visión, misión y valores, aspectos que serán considerados para la próxima actualización de la carrera que se tiene planificada para el futuro inmediato.

\section{Conclusiones}

Después de haber realizado el análisis entre evaluaciones, se considera que el nivel de pertinencia social del programa académico de la MDEE tiene un puntaje de 5.7000 en la escala $0-10$, donde 0 es calidad nula y 10 es excelente la calidad. En la gráfica 1 se aprecia el resultado de la evaluación externa. 


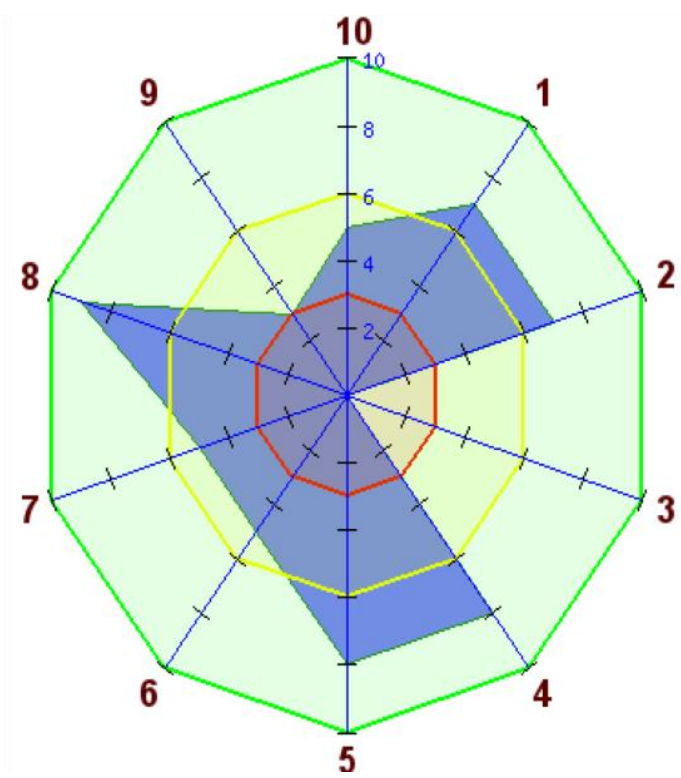

Gráfica 1. Resultados de la evaluación multinacional externa: 1. Congruencia del plan de estudios con los objetivos de la formación, 2. Comparativa del plan de estudios con otros similares, 3. Evaluación y actualización del plan de estudios, 4. Organización y operación del plan de estudios, 5. La investigación vinculada al plan de estudios, 6. Plan de estudios vinculado al posgrado, 7. Contenidos en el plan de estudios sobre cuidados del medio ambiente, uso de energías alternativas, cuidado del aire, agua, equidad de género, etcétera; 8. Las disciplinas relacionadas con el plan de estudios en vinculación con redes temáticas, 9. Vinculación de las tecnologías de la información y la comunicación en el plan de estudios, 10. Servicio social, prácticas profesionales y actividades vinculadas al plan de estudios.

Se sugiere que la MDEE renueve la evaluación (o reevaluación) ante un posible incremento en los niveles de calidad o superación de debilidades siguiendo con el proceso metodológico antes señalado, de acuerdo con el conjunto de actividades planeadas, programadas, con recursos de apoyo y evaluadas, así como las relacionadas con las estrategias utilizadas para superar las debilidades y mantener las fortalezas que el programa educativo habrá de atender con metas claras y concretas. Se recomienda implementar el conjunto de acciones que conforman el plan de mejoramiento a la brevedad que opere de acuerdo con las necesidades detectadas del programa educativo, considerando la estructura 
institucional, las metas a realizar, el cronograma de actividades y los responsables de cada una de ellas.

\section{Bibliografía}

Gacel Ávila, J. (2003). La internacionalización de la educación superior, paradigma de la ciudadanía global. México: Ed. Universidad de Guadalajara. 